\title{
Randomised controlled trial of patient triggered and conventional fast rate ventilation in neonatal respiratory distress syndrome
}

\author{
M W Beresford, N J Shaw, D Manning
}

\begin{abstract}
Aim-To compare patient triggered, with conventional fast rate, ventilation in a randomised controlled trial using the incidence of chronic lung disease as the primary outcome measure.

Methods-Three hundred and eighty six preterm infants with birthweights from 1000 to $2000 \mathrm{~g}$, and requiring ventilation for respiratory distress syndrome within 24 hours of birth, were randomised to receive either conventional or trigger ventilation with the SLE 2000 ventilator.

Results-There were no significant differences in the incidence of chronic lung disease (28 day and 36 week definitions), death, pneumothorax, intraventricular haemorrhage, number of ventilator days, or length of oxygen dependency between groups.

Conclusions-Patient triggered ventilation in preterm infants with respiratory distress syndrome is feasible. No significant differences, when compared with conventional fast rate ventilation in important medium and longer term outcome measures, were evident.

(Arch Dis Child Fetal Neonatal Ed 2000;82:F14-F18)
\end{abstract}

Keywords: patient triggered ventilation; prematurity; respiratory distress syndrome

Despite advances in neonatal practice, mechanical ventilation of infants with respiratory distress syndrome (RDS) may result in the occurrence of a pneumothorax, a factor implicated in the pathogenesis of intraventricular haemorrhage, ${ }^{12}$ or the development of chronic lung disease of prematurity (CLD).$^{13}$ It has been suggested that the use of faster ventilator rates (60-100 breaths/minute) may reduce the incidence of these complications by synchronising the baby's own respiratory rate with that of the ventilator, preventing the infant expiring during the inspiratory phase of the ventilation cycle. ${ }^{4-6}$ An alternative method of synchronising the mechanical ventilator with the infant's own respiratory rate is by using phase and rate matched patient triggered ventilation. ${ }^{7}$ This has been technically possible for several years, owing to the availability of airway pressure or flow sensors that ensure a short trigger response time of the ventilator. ${ }^{89}$ Allowing the infant to trigger a proportion of breaths may also reduce the work of breathing, improve oxygenation, and increase tidal volume. ${ }^{10-12}$ The SLE 2000 ventilator is a valveless jet ven- tilator that operates at an airflow of 5 litres/minute and is capable of inflating the airways in conventional or trigger mode. ${ }^{13}$

Although there are theoretical and potential short term benefits to patient triggered ventilation, ${ }^{7}$ few studies have so far shown any longer term outcome advantages. A study from Plymouth reported the feasibility of using triggered ventilation in 68 infants with RDS. ${ }^{14}$ In this study the authors reported successful treatment with trigger ventilation with no obvious increase in adverse outcomes and a low pneumothorax rate. A more recent, larger study compared synchronised intermittent mandatory ventilation (SIMV), in which many of the infants breaths were triggered, with conventional ventilation in a heterogeneous group of 327 infants. $^{15}$ The results of this study suggested that duration of ventilation and possibly length of oxygen dependency might be reduced in certain birthweight specific groups by using SIMV. Preliminary data from a large, multicentre, randomised comparative trial of patient triggered ventilation and intermittent mandatory ventilation in which a variety of ventilator types were used has been presented. ${ }^{16}$ It suggested, however, no significant differences in important outcomes such as CLD, duration of ventilation, and pneumothorax rate, although complete data are as yet unavailable. There has been no other direct comparison between trigger ventilation and conventional ventilation with respect to medium or longer term outcomes.

This study aimed to perform a prospective, randomised controlled trial comparing a ventilation strategy of trigger ventilation with that of synchronised fast rate conventional ventilation in infants receiving mechanical ventilation for RDS. The primary outcome measure was the incidence of CLD, arguably the most important respiratory outcome of modern neonatal intensive care.

\section{Methods}

The study was performed between April 1993 and October 1998 at the Mersey Regional Neonatal Intensive Care Unit at the Liverpool Women's Hospital (formerly Liverpool Maternity Hospital), Fazakerley Maternity Hospital (25 infants), and Arrowe Park Hospital, Wirral (12 infants). The local research ethics committees of each hospital approved the study.

All infants weighing from 1000 to $2000 \mathrm{~g}$ at birth who received mechanical ventilation within 24 hours of birth with a diagnosis of 
RDS were eligible for inclusion in the study. Respiratory distress syndrome was defined clinically in one of two ways: in an infant not ventilated from birth when, within the first 4 hours, there was sternal retraction, intercostal and subcostal recession, grunting, tachypnoea, hypercapnia and an oxygen requirement above that of room air and a diffuse opacification on the chest radiograph with or without an air bronchogram; in any other infant who at birth had, in the opinion of the attending clinician, sufficient respiratory distress to require intubation and surfactant treatment.

Infants with meconium aspiration syndrome, major malformations, or congenital heart disease were excluded from the study.

TREATMENT ALLOCATION

Once the decision had been made to institute mechanical ventilation, signed parental consent was obtained and the infant's name, unit number, and birthweight were recorded on a standard proforma. Randomisation to the two ventilation strategies was carried out using a computer generated sequence hidden in sequentially numbered, sealed, opaque envelopes. Infants were stratified during randomisation by birthweight (1000 to $1500 \mathrm{~g}$, and 1501 to $2000 \mathrm{~g}$ ). Study design was such as to preclude crossover of treatment strategy. An SLE 2000 ventilator was used for both arms of the study for the entire duration of the study.

\section{TREATMENT SCHEDULES}

After randomisation, the intrinsic respiratory rate of the infant was counted over 30 seconds while the infant was receiving continuous positive airway pressure. In the conventional group, the ventilator rate in breaths/minute was set at, or greater than, the infant's own respiratory rate. In the triggered group the ventilator was switched to trigger mode on the highest sensitivity and a backup rate was set at 20 breaths less than the infant's intrinsic respiratory rate. A rate of 60 breaths/minute was set for both groups if the infant was apnoeic. For both ventilatory modalities, the initial inspiratory to expiratory ratio was 1 to 1 and the inspiratory time set between 0.3 and 0.5 seconds. Peak inspiratory pressure was set between 18 and $24 \mathrm{~cm} \mathrm{H}_{2} \mathrm{O}$, depending on the size of the baby and chest wall movement, and a positive end expiratory pressure of $4 \mathrm{~cm} \mathrm{H}_{2} \mathrm{O}$ was set. The initial inspired oxygen concentration $\left(\mathrm{FIO}_{2}\right)$ was that which provided adequate arterial oxygenation. Inspiratory and expiratory pressures, together with $\mathrm{FIO}_{2}$, were altered at the discretion of the attending clinician in line with blood gases. Throughout the period of ventilation, parameters were set to maintain arterial oxygen tension between 6.67 and 10 $\mathrm{kPa}$ and arterial carbon dioxide tension between 4.67 and $6.0 \mathrm{kPa}$.

In the conventional treatment arm, attempts were made to maintain the ventilator rate to at least that of the infants' respiratory rate. If the infant was constantly expiring during the inspiratory part of the ventilator cycle and there was no improvement in oxygenation a neuromuscular paralysing agent (pancuro- nium) was administered. This was stopped when the infant was weaned to around 30 breaths/minute or to an inspiratory pressure of $20 \mathrm{~cm} \mathrm{H} 2 \mathrm{O}$, or earlier if the physician in charge considered that excessive fluid retention was occurring. The infant was initially weaned from the ventilator by gradually decreasing the peak inspiratory pressure to $12 \mathrm{~cm} \mathrm{H}_{2} \mathrm{O}$ if tolerated, or the lowest pressure attainable above $12 \mathrm{~cm} \mathrm{H} 2 \mathrm{O}$ which did not result in deterioration of blood gases. The ventilatory rate was then reduced, keeping the inspiratory time between 0.3 and 0.5 seconds.

In the triggered ventilation arm of the study, as in the conventional arm, the infant was initially weaned from the ventilator by gradually decreasing the peak inspiratory pressure down to $12 \mathrm{~cm} \mathrm{H}_{2} \mathrm{O}$ if tolerated or the lowest pressure attainable above $12 \mathrm{~cm} \mathrm{H}_{2} \mathrm{O}$, which did not result in deterioration on blood gases. The triggering rate was then weaned using the SIMV mode on the ventilator, starting at an SIMV rate of 20 breaths per minute, keeping the inspiratory time between 0.3 to 0.5 seconds.

Infants in both groups were extubated from a rate of between 5 and 10 breaths/minute. Nasal continuous positive airway pressure either prior to intubation or post extubation was not employed in either ventilatory group.

OUTCOME DEFINITIONS AND TREATMENT POLICIES

Chronic lung disease was defined in two ways: dependency on supplemental oxygen to maintain oxygen saturations at $94 \%$ or above at 28 postnatal days; and dependency on supplemental oxygen to maintain oxygen saturations at $94 \%$ or above at 36 postmenstrual weeks. Pneumothorax was defined as intrathoracic, extra-pulmonary airleak necessitating the insertion of a chest drain. It is the unit's practice to confirm the presence of a patent ductus by Doppler echocardiography before treatment with indomethacin or surgery if this is unsuccessful. Cerebral ultrasound scanning was performed routinely in all infants and the worst grade of intraventricular haemorrhage, together with the presence or absence of periventricular leucomalacia recorded. Intraventricular haemorrhage was defined as follows: grade I echodensity confined to the subependymal region; grade II echodensity extending into the cerebral ventricles, but not the surrounding parenchyma with or without ventricular dilatation; grade III (severe) echodensity extending into the cerebral parenchyma which persisted and resulted in cyst formation.

During the entire period of study, exogenous surfactant treatment was in routine use and it was the policy of the units to administer morphine routinely (infusion or on an as required basis at the discretion of the attending physician) to all preterm infants requiring intubation and mechanical ventilation during the acute phase of their illness. This was in an attempt to reduce agitation, discomfort, and fighting the ventilator, and was withdrawn in the final stage of weaning. Methylxanthines (aminophylline, theophylline, and latterly 
Table 1 Demographic details

\begin{tabular}{|c|c|c|}
\hline & Conventional $n=193$ & Trigger $n=193$ \\
\hline Male (\%) & $112(58)$ & $117(61)$ \\
\hline \multicolumn{3}{|l|}{ Randomisation groups } \\
\hline $1000-1499 \mathrm{~g}$ (\% male) & $131(52)$ & $123(59)$ \\
\hline $1500-1999 \mathrm{~g}$ (\% male) & $62(69)$ & $70(64)$ \\
\hline Gestation weeks (range) & $29(25-34)$ & $29(25-36)$ \\
\hline Birthweight $\mathrm{g}$ (range) & $1320(1006-1996)$ & $1336(1000-1997)$ \\
\hline Antenatal steroids (\%) & $152 / 176(86)$ & $141 / 169(83)$ \\
\hline$\geqslant 2$ doses $(\%)$ & $111 / 176(63)$ & $97 / 169(57)$ \\
\hline \multicolumn{3}{|l|}{ Mode/reason of delivery (\%) } \\
\hline $\mathrm{EmCS}-\mathrm{PIH} / \mathrm{APH}$ & $29(15)$ & $47(24)$ \\
\hline EmCS-fetal distress & $43(22)$ & $55(28)$ \\
\hline Spontaneous vaginal delivery & $70(36)$ & $55(28)$ \\
\hline Semi-elective CS-IUGR & $7(4)$ & $2(1)$ \\
\hline Other/not apparent & $44(23)$ & $34(18)$ \\
\hline \multicolumn{3}{|l|}{ Sepsis (\%) } \\
\hline Any septic episode & $66(34)$ & $61(32)$ \\
\hline Total blood culture positive episodes & 87 & 78 \\
\hline CNS positive episodes & 81 & 70 \\
\hline$>1$ CNS positive episodes & $15(8)$ & $15(8)$ \\
\hline Not known & $17(9)$ & $24(12)$ \\
\hline
\end{tabular}

EmCS = emergency caesarean section; $\mathrm{PIH}=$ pregnancy induced hypertension; $\mathrm{APH}=$ antepartum haemorrhage; IUGR = intrauterine growth retardation; CNS = coagulase negative staphylococci

Table 2 Patient treatment characteristics

\begin{tabular}{lll}
\hline & Conventional & Trigger \\
\hline Surfactant doses: median (range) & $2(0-3)$ & $2(0-4)$ \\
Artificial surfactant (\%) & $185(96)$ & $189(98)$ \\
Neuromuscular paralysis (\%) & $12 / 193(6)$ & $12 / 193(6)$ \\
Diuretics (\%) & $12 / 175(7)$ & $13 / 169(8)$ \\
Postnatal steroids (\%) & $14 / 175(8)$ & $12 / 169(7)$ \\
\hline
\end{tabular}

caffeine) were used throughout the study period in accordance with unit guidelines in all infants less than 35 weeks of gestation at risk of apnoea of prematurity. This was begun in the final phase of weaning when the breaths/ minute were approximately 20 /minute.

STATISTICAL CONSIDERATIONS

The incidence of CLD (supplementary oxygen requirement at 28 days) between 1991 and 1993 at Liverpool Maternity Hospital in infants weighing from 1000 to $2000 \mathrm{~g}$ who received mechanical ventilation was around $20 \%$. It was calculated that to detect a $50 \%$ reduction in the incidence of CLD $(\alpha 0.05, \beta=$ 0.2) 193 infants would be required in each treatment group. Statistical analyses including $\chi^{2}$ test, Mann Whitney $U$ test, odds ratio (OR) and $95 \%$ confidence intervals (95\% CI) were performed using the Arcus Quickstat Biomedical Version 1.0 statistical package.

\section{Results}

Three hundred and eighty six infants were enrolled in the study; 193 were randomly allocated to conventional ventilation and 193 to patient triggered ventilation. The median time

Table 3 Secondary outcome data

\begin{tabular}{llll}
\hline & $\begin{array}{l}\text { Conventional } \\
n=193\end{array}$ & Trigger $n=193$ & Odds ratio (95\% CI) \\
\hline $\begin{array}{l}\text { Death before 28 days (\%) } \\
\text { Death before 36 weeks (\%) }\end{array}$ & $7(3.6)$ & $15(7.8)$ & $0.45(0.15-1.20)$ \\
$\begin{array}{l}\text { Pneumothorax (\%) } \\
\text { IVH total population }\end{array}$ & $21(1.1)$ & $15(7.8)$ & $0.51(0.18-1.33)$ \\
$\quad$ Grade III (\%) & $13(7)$ & $20(10)$ & $1.06(0.52-2.13)$ \\
$\quad$ Grade III IVH/death (\%) & $20(10)$ & $11(6)$ & $1.19(0.48-3.03)$ \\
IVH survivors & $12(6)$ & $7(11)$ & $0.90(0.48-1.80)$ \\
$\quad$ Grade III (\%) & $12(6)$ & $8(5)$ & $1.69(0.60-5.20)$ \\
Cystic PVL survivors (\%) & $11(6)$ & $5(3)$ & $1.47(0.54-4.26)$ \\
Shunt insertion survivors (\%) & & $2.19(0.68-8.19)$ \\
\hline
\end{tabular}

$\mathrm{IVH}=$ intraventricular haemorrhage; $\mathrm{PVL}=$ periventricular leucomalacia; of entry into the study was 2 hours (range 0-24 hours) for the conventional group and 2 hours (range 0-24 hours) for the trigger group.

Table 1 shows the demographic data of infants randomised to the two study groups. Postnatal treatment characteristics are shown in table 2 and showed no significant difference between the groups.

Thirty one per cent (58/186) of the survivors in the conventional group developed CLD, defined as oxygen dependency at 28 days compared with $32 \%(57 / 178)$ of survivors in the trigger group (OR 0.96; 95\% CI 0.60-1.53; $\mathrm{p}=0.95)$. At 36 weeks of postmenstrual age the incidence of oxygen dependency among survivors was $29 \%(53 / 185)$ and $32 \%$ (57/178), respectively (OR 0.85 ; 95\% CI 0.53-1.37; $\mathrm{p}=0.56)$. Thirty two per cent $(61 / 193)$ in the conventional group died or had CLD at 36 weeks of postmenstrual age compared with $37 \%(72 / 193)$ in the trigger group (OR 0.78; 95\% CI $0.50-1.21 ; \mathrm{p}=0.28)$.

Table 3 shows secondary outcome measures for each group. None of these differences was significant. Among survivors, the median number of days of continuous ventilation for the conventional group was four days (range 1 to 150) and the triggered group three days (range 1 to $42 ; \mathrm{p}=0.19$ ). The median number of days among survivors who required supplemental oxygen was five (range 0-150) in the conventional arm and four days (range $0-159$ ) in the triggered $\operatorname{arm}(p=0.30)$.

The incidence of patent ductus arteriosus requiring treatment with indomethacin and/or surgical ligation is shown in table 4. Although the requirement for individual treatments alone showed no significance difference between groups, the incidence of patent ductus requiring indomethacin and/or ligation was higher in the conventional group for both survivors $(p=0.047)$ and the whole population of infants $(\mathrm{p}=0.04)$.

Twenty five infants (13\%) in the trigger group were deemed by the supervising clinician to have failed on this mode of ventilation and changed to an alternative mode of ventilation. Twenty four of these received conventional ventilation and one high frequency oscillatory ventilation. No other infant in the trial received high frequency oscillation. Reasons for failing were: persisting severe respiratory acidosis or inadequate oxygenation requiring high peak inspiratory pressure and/or paralysis (14 infants); poor respiratory effort and/or need for bag and mask resuscitation for desaturating episodes (4 infants); specific reason for clinician changing not apparent (9 infants). There was a significant difference in the median gestational age of those failing on trigger compared to those not failing (trigger failures 28 weeks, range 26-32; non-failures 29 weeks, range $25-36 ; p=0.02)$. This was not the case for the median birthweight between failed and non-failed trigger infants (trigger failures 1246 g, range 1000-1988 g; non failures $1343 \mathrm{~g}$, range $1000-1997 \mathrm{~g} ; \mathrm{p}=0.24$ ).

The median gestational age of all those dying was significantly lower than those surviving (dying 28 weeks, range 26-34 weeks; surviving 
Table 4 Incidence of patent ductus arteriosus

\begin{tabular}{lccc}
\hline & Conventional & Trigger & Odds ratio (95\% CI) \\
\hline Total population & 193 & 93 & \\
$\quad$ Indomethacin only & 25 & 14 & $1.90(0.91-4.09)$ \\
Ligation & 8 & 4 & $2.04(0.53-9.42)$ \\
Indomethacin +/- ligation (\%) & $33(17)$ & $18(9)$ & $2.01(1.05-3.93)$ \\
Survivors & 185 & 178 & \\
$\quad$ Indomethacin only & 25 & 13 & $1.98(0.94-4.37)$ \\
Ligation & 7 & 4 & $1.71(0.43-8.10)$ \\
Indomethacin +/- ligation (\%) & $32(17)$ & $17(10)$ & $1.98(1.02-3.96)$ \\
\hline
\end{tabular}

29 weeks, range $25-36$ weeks; $p=0.03$ ), but there was no significant difference in birthweight between all those dying and survivors (dying: 1246 g, range 1010-1924; survivors 1340, range $1000-1997 ; \mathrm{p}=0.06)$. There was, however, no significant difference between those dying and those surviving in the median gestational age for either group (conventional, dying: 28 weeks, range 26-34; surviving: 29 weeks, range $25-34, \mathrm{p}=0.25$; trigger, dying: 28 weeks, range 26-32; surviving: 29 weeks, range $25-36, p=0.053)$ or in their median birthweight (conventional, dying: $1254 \mathrm{~g}$, range 1036-1924; surviving: $1330 \mathrm{~g}$, range 1000 1996, $\mathrm{p}=0.43$; trigger, dying: $1246 \mathrm{~g}$, range 1010-1670; surviving: $1343 \mathrm{~g}$, range 1000 1997, $\mathrm{p}=0.06$ ).

\section{Discussion}

We have presented a large randomised controlled trial comparing the medium and longer term outcomes of two different strategies of ventilation for preterm infants with RDSpatient triggered ventilation and conventional fast rate ventilation. Both strategies aim to ensure synchrony of the infants breathing with the ventilator breaths. This was a pragmatic clinical trial: randomised infants were therefore analysed on an intention to treat basis and no crossover was allowed, although a small number of infants randomised to trigger were eventually ventilated according to standard guidelines, usually fast rate conventional ventilation.

Importantly, a single ventilator was used for both arms of the trial across all three units. Although infants weighing under $1000 \mathrm{~g}$ have been reported to successfully generate triggered breaths with the SLE 2000, ${ }^{14}$ other work, ${ }^{7}$ together with our own experience, has suggested that these infants may not do this consistently, leading to most breaths being provided by the mandatory backup rate. Infants weighing more than $2000 \mathrm{~g}$ are at lower risk of CLD. ${ }^{3}$ For these reasons we randomised only infants weighing from 1000 to $2000 \mathrm{~g}$ at birth.

The difference in incidence of CLD between groups (our primary outcome measure) was not significant, using either definition of oxygen dependency -28 postnatal days or 36 postmenstrual weeks. Our sample size calculation, looking for a $50 \%$ reduction in this outcome, allowed a large yet locally manageable study to be performed. Local practice was therefore consistent between contributing units. A smaller, perhaps clinically important difference of, for example, $20 \%$ reduction in incidence of CLD, would have required a sample size of almost 3000 infants based on our historical incidence. Preliminary data from a four year, international, multicentre comparative trial recruited considerably fewer than this (927 infants). ${ }^{16}$ It is extremely unlikely that the difference in incidence of oxygen dependency at 36 weeks of postmenstrual age between our treatment groups would become significant even if considerably more babies had been recruited (sample size around 7500 infants). Differences requiring a sample size of this magnitude may only be shown to be significant using meta-analysis. Confidence intervals for this and the other outcome measures allow us to be aware of the range of difference that can be excluded by our study. Chronic lung disease (oxygen requirement at 36 weeks) showed a conventional to trigger odds ratio of 0.85 with $95 \%$ confidence intervals 0.53 to 1.37 . Although we can not say the two strategies will have exactly the same incidence of CLD, it is unlikely that conventional ventilation causes an increase in CLD of more than $37 \%$ or that it causes a decrease of more than $47 \%$.

The sample size for the trial was estimated on the previous two years' experience of the incidence of CLD in babies of the same weight range (1000-2000 g) who were to be studied. The actual incidence of CLD in both arms of the trial was somewhat higher than this indicating the difficulties inherent in estimating sample sizes based on historical data.

This was a clinical study aimed to provide information that might enable guidelines to be optimised for neonatal ventilation. We therefore used a clinical definition of RDS that was easily applied and likely to be accepted in most units. In both treatment groups the inspiratory time was maintained between $0.3-0.5$ seconds which provided an adequate tidal volume, but reduced the possibility of the infant expiring against the inspiratory phase of the ventilatory cycle. ${ }^{7}$ We chose a priori to wean the trigger infants using the SIMV mode. Other workers have weaned their infants receiving trigger ventilation by reducing the peak inspiratory pressure down to $8 \mathrm{~cm} \mathrm{H}_{2} \mathrm{O} \cdot{ }^{14}$ Our experience of this approach has not been successful, the lower peak inspiratory pressure used being associated with a deterioration of blood gases. The advantage of using SIMV mode during the weaning phase permits progressive reduction in the rate of ventilator support right up to the point of extubation ${ }^{7}$ and initial studies confirmed no marked difference between these regimens. ${ }^{17}$ We therefore empirically decided to use the SIMV mode for weaning. More recent work comparing weaning using patient triggered ventilation with SIMV in 20 infants showed no significant differences in duration of weaning and failure of weaning for an SIMV rate down to 20 breaths/minute. However, it did indicate there might be a shorter duration of weaning for those receiving patient triggered ventilation compared with SIMV when the SIMV rate was reduced to 5 to 10 breaths/minute. ${ }^{18} \mathrm{~A}$ recent review of the patient triggered ventilation, however, suggests the best mode for weaning during patient triggered ventilation remains unresolved. ${ }^{19}$

Our patient groups were well matched for sex, gestational age, birthweight and use of 
antenatal steroids. Treatment with surfactant, diuretics and postnatal steroids were similar between groups, as was the incidence of sepsis. The number of infants in the conventional arm that were paralysed was low compared with a previous study from one of the units involved in the trial. ${ }^{6}$ This probably reflects nursing and medical staff being more comfortable with the conventional faster rate ventilation in more recent years. The incidence of pneumothorax in each group was the same and is similar to the incidence of pneumothorax previously reported from Liverpool Maternity Hospital in the post surfactant era. ${ }^{6}$ This incidence seems to be greater than that in the previous descriptive study of trigger ventilation. ${ }^{14}$ However, several infants in the latter study sustained a pneumothorax before receiving mechanical ventilation and if these are included in the overall figures in this study the incidence of pneumothorax is similar to ours. The incidence of patent ductus arteriosus receiving treatment with indomethacin or ligation was higher in the conventional group. There is no clear explanation for this. Increased hypoxia in the conventional group might have predisposed them to a higher risk of patent ductus arteriosus, although length of ventilation in each group and length of oxygen dependency were similar.

We were not able to support the suggestion from a previous study that trigger ventilation reduces the length of oxygen dependency, or duration of ventilation. ${ }^{15}$ None of our infants received trigger ventilation from birth, although all did within the first 24 hours. It might be argued that starting trigger ventilation earlier could have reduced the risk of pneumothorax and possibly CLD. Our study is unable to answer this, but we feel an effect on these outcomes is unlikely, as the median time of entry into the study was two hours.

Infants receiving trigger ventilation, although deemed by the attending clinician to have failed for various reasons, showed a significantly lower median gestational age than the non-failure infants. This is despite them having a birthweight of $1000 \mathrm{~g}$ or greater and supports data that suggest gestational age could be an important determinant in selecting infants for trigger ventilation..$^{20}$ There was not, however, a significant difference in gestational age or birthweight between those dying and those surviving within either the conventional or trigger group.

In conclusion, we have shown the feasibility of ventilating preterm infants with RDS using patient triggered ventilation. No significant differences in important medium to longer term outcome measures were shown when comparing it with conventional fast rate ventilation. A much larger study is necessary to detect smaller differences in these outcomes.

We acknowledge the help of Dr J Robertson for entering patients into the study at Arrowe Park Hospital; Professor RWI Cooke, Professor AM Weindling, Dr WC Yoxall and Dr NV Subhedar for allowing us to enter their patients at Liverpool Women's Hospital into the study.

1 Ramsden CA, Reynolds EOR. Ventilator settings for newborn infants. Arch Dis Child 1987;62:529-38.

2 Greenough A, Wood S, Morely CJ, Davis JA. Pancuronium prevents pneumothoraces in ventilated premature babies who actively expire against positive pressure ventilation. who actively expire

3 Shaw NJ, Gill B, Weindling AMW, Cooke RWI. The changing incidence of chronic lung disease. Health Trends 1993;25:50-3.

4 South M, Morley CJ. Synchronous mechanical ventilation of the neonate. Arch Dis Child 1986;61:1190-5.

5 Field D, Milner AD, Hopkin IE. Manipulation of ventilator settings to prevent active expiration against positive pressure inflation. Arch Dis Child 1985;60:1036-40.

6 Shaw NJ, Cooke RWI, Gill AB, Shaw NJ, Saeed M. Randomised trial of routine versus selective paralysis during ventilation for neonatal respiratory distress syndrome. Arch Dis Child 1993;69:479-82.

7 Greenough A, Milner AD. Respiratory support using patient triggered ventilation in the neonatal period. Arch Dis Child 1992;67:69-71.

8 Hird MF, Greenough A. Comparison of triggering systems for neonatal patient triggered ventilation. Arch Dis Child 1991;66:426-8.

9 Greenough A, Greenall F. Patient triggered ventilation in premature neonates. Arch Dis Child 1988;63:77-8.

10 Cleary JP, Bernstein G, Heldt GP, Mannino FL. Improved oxygenation during synchronised intermittent mandatory
ventilation in neonates with respiratory distress syndrome: a randomised crossover study. $\mathcal{F}$ Pediatr 1995;126:407-11.

11 Bernstein G, Heldt GP, Mannino FL. Increased and more consistent tidal volumes during synchronised intermittent mandatory ventilation in new-born infants. Am F Respir mandatory ventilation in new-bor
Cri Care Med 1994;150:1444-8.

12 Rosas FR, Cleary JP, Mannino FL,Bernstein G, Heldt GP. Decreased diaphragmatic effort and increased mechanical ventilator efficiency in rabbits on synchronised IMV. Am $\mathcal{F}$ Respir Crit Care Med 1995;151:A435.

13 Chan KN, Chakrabati MK, Whitwam JG, Silverman M. Assessment of a new valveless infant ventilator. Arch Dis Child 1988;63:162-7.

14 de Boer RC, Jones A, Ward PS, Baumer JH. Long term trigger ventilation in neonatal respiratory distress syndrome. Arch Dis Child 1993;68:308-11.

15 Bernstein G, Mannino FL, Heldt GP, et al. Randomised multicenter trial comparing synchronised and conventional intermittent mandatory ventilation in neonates. 7 Pediatr 1996;128:453-63.

16 Baumer JH, Wright D, Mill T. Patient triggered ventilation compared to intermittent mandatory ventilation for neonatal respiratory distress syndrome: an open multicentre randomised controlled trial. Proceedings of the $2^{n d}$ Annual Spring meeting. London: Royal College of Paediatrics and Child Health, 1998: Abstract G74.

17 Chan V, Greenough A. Comparison of weaning by patient triggered ventilation or synchronous intermittent mandatory ventilation in preterm infants. Acta Pediatr tory ventilation

18 Dimitriou G, Greenough A, Giffin F, Chan V. Synchronous intermittent mandatory ventilation modes compared with patient triggered ventilation
Child 1995;72:F188-F90.

19 Donn SM, Sinha SK. Controversies in patient triggered ventilation. Clin Perinatol 1998;25:49-61.

20 Mitchell A, Greenough A, Hird M. Limitations of patient triggered ventilation in neonates. Arch Dis Child 1989;64:924-9. 